\title{
Physikalische Blätter
}

30. Jahrgang · Mai 1974 · Heft 5

\section{Sonderdruck}

(C) 1974 Physik Verlag GmbH, Weinheim/Bergstr.

Bei der Wiedergabe von Gebrauchsnamen, Handelsnamen, Warenbezeichnungen und dgl. handelt es sich häufig um gesetzlich geschützte eingetragene Warenzeichen, auch wenn sie nicht als solche mit ${ }^{\circledR}$ gekennzeichnet sind. 



\section{Speicherung von Elektronen und Positronen im DORIS beim DESY}

\section{Von Helmut Wiedemann, Hamburg*)}

\section{Stand des Speicherringes DORIS}

Nach mehrjähriger Bauzeit konnten am 17. Dezember 1973 endlich die ersten Versuche gestartet werden, Elektronen in einen Ring des Doppelspeicherringes DORIS einzuschießen und sie dort zu speichern (s. auch Physik. Bl. 30 [1974] Nr. 1, S. 42).

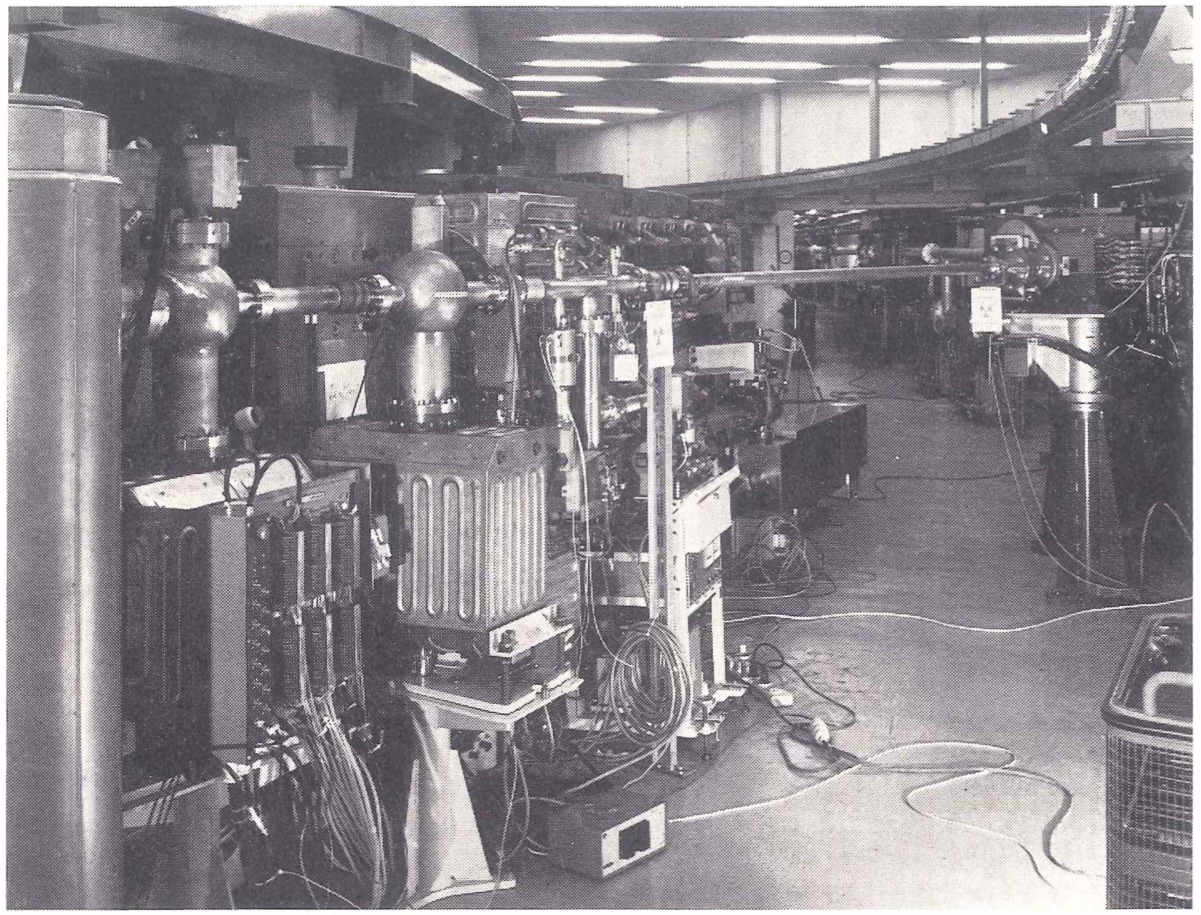

Bild 1. Zuführung des Elektronenstrahles vom Synchrotron (Rohrsystem oberhalb der Bildmitte) und Einschuß in den Speicherring (links).

Ein Speicherring ist eines der modernsten Geräte der Hochenergiephysik. Im Gegensatz zu einem Synchrotron, in dem die Elektronen nur beschleunigt und dann dem Experiment zugeführt werden, sind in einem Speicherring zwei Teilchenstrahlen, die in entgegengesetzter Flugrichtung umlaufen, für mehrere Stunden mit gleicher Energie gespeichert. In den sog. Wechselwirkungspunkten durchkreuzen sich diese Strahlen und können durch Zusammenstoß einen Prozeß auslösen, der vom Hochenergiephysiker beobachtet und analysiert wird.

*) Dr. Helmut Wiedemann, Deutsches Elektronen-Synchrotron (DESY), 2 Hamburg 52, Notkestieg 1. 
Nachdem bei den ersten Einschußversuchen die Elektronen zunächst schon kurz nach dem Einschußpunkt im Speicherring verlorengingen, konnte der Elektronenstrahl durch geeignete Wahl der Korrekturen am magnetischen Führungsfeld wenig später einen, mehrere und dann einige hundert Umläufe überleben. Nicht lange sollte es danach dauern, bis die Elektronen im Speicherring auch akkumuliert werden konnten. Dies ist ein besonderes Charakteristikum eines Speicherrings gegenüber einem Synchrotron. Während für ein Synchrotron ein Injektionspuls pro Beschleunigungszyklus genügt, sind in einem Speicherring zu einem sinnvollen Betrieb viele Injektionspulse nötig, bis die notwendige Strahlenintensität erreicht ist. Der Grund ist klar: Die Anzahl der hochenergetischen Prozesse ist proportional der Intensität des hochenergetischen Strahles und der Dichte des Targets, auf das dieser Strahl gerichtet wird. Dieses Target ist im Falle eines Synchrotrons ein fester Körper oder eine Flüssigkeit, d. h. die Dichte ist sehr hoch und es genügt eine relativ geringe Strahlintensität, um die gewünschte Häufigkeit an hochenergetischen Prozessen zu erreichen. Ganz anders liegen diese Parameter in einem Speicherring. Hier ist der eine Strahl das Target für den anderen Strahl. Es müssen also höhere Strahlintensitäten erreicht werden als in einem Synchrotron.

In den ersten sechs Wochen der Inbetriebnahme von DORIS ist es gelungen, Elektronenströme bis zu $320 \mathrm{~mA}$ und Positronenströme bis zu $170 \mathrm{~mA}$ zu speichern. Das Hauptaugenmerk für die weitere Verbesserung von DORIS wird sein, die Effekte, die diese maximalen Ströme begrenzen, zu studieren und gezielt zu bekämpfen. Ziel wird es sein, in beiden Strahlen eine Teilchenintensität zu erreichen, die einem umlaufenden Strom von je etwa einem Ampere entspricht.

In den folgenden Abschnitten werden die großen Komponenten des Speicherringes und ihre Wirkungsweise diskutiert werden.

\section{Magnetstruktur}

Die augenfälligste Großkomponente eines Speicherringes ist das Magnetsystem, bestehend aus Ablenkmagneten, Quadrupolen, Sextupolen und Oktupolen. Im DORIS sind alle Magnete und auch die anderen Großkomponenten wie Vakuum, Hochfrequenz, Strahlbeobachtung usw. doppelt vorhanden. Wie der Name sagt, besteht DORIS - Doppel-Ring-Speicher - aus zwei Speicherringen, je einem für die Elektronen und für die Positronen. An zwei Punkten, den Wechselwirkungspunkten, durchkreuzen sich beide Strahlen. Mit dieser vollständigen Trennung beider Strahlen wurde eine individuelle Strahlkorrektur erreicht, was den Betrieb des Speicherringes sehr erleichtert. Außerdem können höhere Strahlintensitäten gespeichert werden als in einem Speicherring mit nicht getrennten Strahlen. Die Funktion der Ablenkmagnete ist ganz offensichtlich nur die, eine mehr oder weniger kreisförmige geschlossene Umlaufbahn für die Elektronen und Positronen zu erzwingen.

Die Quadrupolmagnete dienen der Fokussierung der Teilchenstrahlen. Wenn es in der Lichtoptik selbst mit Hilfe der Lasertechnik nicht möglich ist, einen parallelen Strahl zu erzeugen, der auf einer Strecke von einer Milliarde Kilometern - dies ist die Flugstrecke der Elektronen oder Positronen im Speicherring während einer Speicherzeit von einer Stunde - nur unmerklich dicker wird, so ist dies für einen Teilchenstrahl völlig illusorisch. Es werden also Lin- 
sen benötigt, nämlich die Quadrupolmagnete, die den auseinanderlaufenden Strahl immer wieder fokussieren. Die Gesamtzahl der Quadrupole in einem Ring definiert für die Teilchenstrahlen eine Potentialmulde, in der die Teilchen transversal, d. h. horizontal und vertikal, stabile Schwingungen um eine Gleichgewichtslage ausführen. Diese Schwingungen nennt man Betatronschwingungen. Wie jede Schwingung sind auch die Betatronschwingungen durch das Einwirken von Störfeldern, wie etwa das Erdfeld, Streufelder von Vakuumpumpen, Fehlaufstellungen von Magneten usw., linearen und nichtlinearen Resonanzerscheinungen unterworfen. Durch geeignete Wahl der Fokussierung, d. h. der Tiefe der Potentialmulde, muß darauf geachtet werden, daß sich solche Resonanzerscheinungen nicht störend bemerkbar machen.

Wie bei der Lichtoptik gibt es auch bei der Teilchenoptik eine chromatische Aberration. Leider ist es nicht möglich, eine Speicherringoptik völlig achromatisch zu machen. Mit Hilfe von Sextupolfeldern läßt sich jedoch erreichen, daß die Betatronschwingungsfrequenz unabhängig von der Teilchenenergie wird. Diese Korrektur hat sich bei allen Speicherringen als notwendig erwiesen, um höhere Strahlintensitäten speichern zu können.

Sollte trotz aller Vorsicht bei der Wahl der Betatronschwingungsfrequenz. und trotz Korrektur der chromatischen Effekte ein resonanzartiges Ansteigen der Schwingungsamplitude auftreten, so können die Oktupolmagnete eine letzte Rettung zur Stabilisierung der Strahlen darstellen. Oktupole bewirken eine: quadratische Anderung der Betatronschwingungsfrequenz mit der Amplitude; d. h. die Schwingungsfrequenz des Teilchens wird von der Anregungsfrequenz weggeschoben, so daß ein weiteres Anwachsen der Schwingungsamplitude verhindert wird.

\section{Das Hochfirequenzsystem}

Die Ablenkung der Teilchenstrahlen in den Ablenkmagneten veranlaßt die Teilchen zur Aussendung elektromagnetischer Wellen, der sog. Synchrotronstrahlung. Die dadurch verlorene Energie muß den Teilchen in Beschleunigungsstrecken, welche die Form von Hochfrequenzresonatoren haben, wieder zugeführt werden. Dieser Energieverlust kann recht hoch sein; im Falle von DORIS ist er im Mittel 1,1 MeV bei einer Teilchenenergie von 3,5 GeV. Die integrierte elektrische Feldstärke in den Beschleunigungsstrecken muß demnach auch mindestens $1,1 \mathrm{MeV}$ sein. In Wirklichkeit muß diese Spannung sogar um den Faktor 2-3 größer sein. Der Grund dafür liegt in der quantenhaften Aussendung des Synchrotronlichtes. Die Teilchen von 3,5 GeV verlieren zwar im Mittel nur 1,1 MeV pro Umlauf, können aber auch mal wesentlich mehr Energie verlieren. In diesen Fällen verhindert die 2-3mal größere Beschleunigungsspannung, daß ein solches Teilchen verlorengeht. Nach einigen tausend Umläufen ist ein solcher allzu großer Energieverlust wieder ausgeglichen.

Das Beispiel sagt uns auch etwas über die gesamte Synchrotronstrahlungsleistung und damit über die Leistung des Hochfrequenzsystems. Wollten wir ein Ampere bei 3,5 GeV im DORIS speichern, würde die dem Strahl zuzuführende Hochfrequenzdauerstrichleistung 1,1 MW pro Strahl sein. Im DORIS beträgt die installierte Hochfrequenzleistung $750 \mathrm{~kW}$ pro Strahl. Es kann also bei 3,5 GeV nur ein Strahlstrom von 600 bis $700 \mathrm{~mA}$ gespeichert werden. Da der Energieverlust pro Umlauf mit der vierten Potenz der Teilchen- 
energie variiert, können also bei niederen Energien weit höhere Ströme und bei höheren Energien nur noch kleinere Ströme gespeichert werden.

\section{Das Vakuumsystem}

Die gespeicherten Teilchenstrahlen sollen eine Lebensdauer von mehr als einer Stunde haben; in dieser Zeit werden die Hochenergie-Experimente durchgeführt. Um solch eine lange Lebensdauer zu erreichen, müssen Kollisionen der Teilchen mit Gasatomen weitgehend ausgeschlossen werden. Dies ist in dem erforderlichen Maße nur in einem Ultrahochvakuum möglich. Der Gasdruck muß kleiner als 10-8 Torr sein. Dieser Gasdruck kann relativ einfach unterschritten werden, falls kein Strahl gespeichert ist. Wird jedoch ein Strahl gespeichert, so trifft die Synchrotronstrahlung auf die Vakuumkammerwand. Durch die starke lokale Erhitzung diffundiert Gas aus dem Material der Vakuumkammer, wodurch der Gasdruck stark erhöht wird.

Ein weiteres Problem, das mit dem Vakuum zusammenhängt, bilden die Ionen der Resigasatome, die durch die hochenergetischen Strahlen erzeugt werden. Diese Ionen werden im elektrostatischen Potential des Elektronenstrahles gehalten, da ihre thermische Energie zu gering ist, um aus diesem Potential zu entweichen. Diese Ionenwolke stellt eine zusätzliche Druckerhöhung im Bereich des gespeicherten Elektronenstrahles dar und verkürzt dadurch zusätzlich die Lebensdauer dieses Strahles. Daneben kann diese Ionenwolke in Schwingungen geraten und durch Rückwirkung auf den Strahl diesen instabil werden lassen. Es müssen also transversale elektrische Felder dafür sorgen, daß diese Ionen aus dem Strahl abgesaugt werden. Dieses Absaugen ist natürlich beim Positronenstrahl nicht nötig, da die positiv geladenen Ionen von den Positronen abgestoßen werden.

\section{Strahllbeobachtung}

Notwendige Hilfsmittel beim Betrieb eines Speicherringes sind die Strahlbeobachtungsinstrumente. Dies ist insbesondere ein Gerät zur Messung des Strahlstromes, das nach der Wirkungsweise einer Förstersonde das intensitätsabhängige Magnetfeld des Strahles mißt. Ein einwandfreies Funktionieren eines Speicherringes hängt sehr davon ab, wie gut die wirkliche Lage des Strahles mit der berechneten Sollage übereinstimmt. Dies wird mit den Positionsmonitoren festgestellt, die in kürzeren Abständen auf dem Umfang des Speicherringes verteilt sind. Dazu wird die durch die Hochfrequenz verursachte zeitliche Struktur des Strahles ausgenutzt. Da beim DORIS die Hochfrequenz 500 $\mathrm{MHz}$ beträgt, sind die Teilchen in Bündeln im Abstand von $60 \mathrm{~cm}$ gleichmäßig über den Umfang verteilt. Bringt man eine kleine Drahtschleife in die Nähe des Strahls, so wird von dem Strahl in dieser Schleife ein hochfrequentes Signal erzeugt. Die Amplitude eines solchen Signals ist vom Abstand zwischen Strahl und Schleife abhängig. Hat man Schleifen auf beiden Seiten des Strahles, so bildet das Differenzsignal ein $\mathrm{Ma}$ für die Abweichung des Strahles vom Mittelpunkt zwischen den beiden Schleifen. Mit Hilfe von Korrekturmagnetfeldern kann die Lage des Strahles auf die gewünschte Mittellage gebracht werden.

Zur Erzielung einer hohen Ereignisrate ist eine hohe Strahldichte erforderlich. Diese Dichte kann durch Vermessen der Intensität des Synchrotronlichtes quer zum Strahl ermittelt werden. 


\section{Kontrolle}

Ein Speicherring stellt ein sehr hoch komplexes System dar, dessen Bedienung die Hilfe eines Prozeßrechners erforderlich macht. Dieser Rechner wird insbesondere zur Berechnung der Stärken der verschiedenen Magnettypen herangezogen, er wird weiter diese Magnetstärken an den Stromversorgungsgeräten einstellen und eine simultane Veränderung dieser Magnetstärken vornehmen, falls z. B. die Energie verändert oder die Fokussierungseigenschaften des Speicherringes geändert werden sollen. Daneben gibt es eine große Anzahl von Uberwachungs- und Regelungsaufgaben.

\section{Experimentelle Aufbauten}

Die besondere Erzeugung von hochenergetischen Ereignissen in Speicherringen erfordert spezielle Anordnungen, um diese Ereignisse zu analysieren. Da bei kollidierenden Elektronen- und Positronenstrahlen ihr Schwerpunktsystem im Wechselwirkungspunkt ruht, fliegen die Produkte hochenergetischer Prozesse im allgemeinen in einem sehr großen Raumwinkel auseinander. Die ideale experimentelle Anordnung wird also in einem Raumwinkel von $4 \pi$ um den Wechselwirkungspunkt alle Reaktionsteilchen erfassen können. Erfassen heißt dabei, daß die Bahn und die Energie aller Teilchen gemessen wird. Die Bahn, aus der die Winkel zu einer Referenzachse bestimmt werden, kann z. B. in Funkenkammern fotografiert werden. Ihre Energie kann aus der Krümmung der Teilchenbahn in einem bekannten Magnetfeld berechnet werden. Darüber hinaus muß die Teilchenart ermittelt werden, was mit Hilfe von Absorbern verschiedener Dicke geschehen kann.

Um den Wechselwirkungspunkt wird also eine Vielzahl von Szintillationszählern und Funkenkammern in einem starken Magnetfeld angeordnet sein, die wiederum von vielen Tonnen Absorbern umgeben sind.

Beim DORIS sind die Aufgaben dieses idealen $4 \pi$-Detektors in zwei Detektoren aufgeteilt worden. An einem Wechselwirkungspunkt wird das Magnetfeld eines supraleitenden Soleonids (PLUTO) die Erfassung fast aller Teilchen in einem großen Raumwinkel mit nicht allzu großer Impulsauflösung erlauben. Im anderen Wechselwirkungspunkt wird ein Doppelarm-Spektrometer (DASP) aufgebaut, das in einem Raumwinkel von nur etwa $1 \pi$ die Impulse der T'eilchen mit großer Auflösung bestimmt. Beide Geräte zusammen ergänzen sich und werden dem Hochenergiephysiker die Möglichkeit geben, tiefer in die Geheimnisse der Natur einzudringen. 



\section{Physikalische Blätter}

30. Jahrgang ' Mai 1974 · Heft 5

Sonderdruck

(C) 1974 Physik Verlag GmbH, Weinheim/Bergstr.

Bei der Wiedergabe von Gebrauchsnamen, Handelsma gen, renbezeichnungen und dgl. handelt es sich häufig uTh geschützte eingetragene Warenzeichen, auch wenn sice solche mit ${ }^{\circledR}$ gekennzeichnet sind. 



\section{Physikalische Blätter}

30. Jahrgang · Mai 1974 ' Heft 5

\section{Sonderdruck}

(c) 1974 Physik Verlag GmbH, Weinheim/Bergs t -

Delsnamen, WaBei der Wiedergabe von Gebrauchsnamen, $\mathrm{H}$. I renbezeichnungen und dgl. handelt es sich hall geschützte eingetragene Warenzeichen, auch solche mit ${ }^{\circledR}$ gekennzeichnet sind. 
